\title{
Transaction
}

\section{The Effect of Applied Voltage and Laser Power on the Molecular Orientation of Laser-Electrospun Poly(ethylene terephthalate) Fibers}

\author{
Kazuhiro Nakata $^{* 1}$, Shinya Kinugawa ${ }^{* 1}$, Midori Takasaki ${ }^{* 2}$, Yutaka Ohkoshi ${ }^{* 1}$, Yasuo Gotoh ${ }^{* 1}$, \\ and Masanobu Nagura* ${ }^{* 1}$
}

\author{
${ }^{* 1}$ Faculty of Textile Science of Technology, Shinshu University, \\ ${ }^{* 2}$ Satellite Venture Business Laboratory, Shinshu University, 3-15-1, Tokida, Ueda, Nagano 386-8567, Japan
}

\begin{abstract}
Laser-electrospinning is a melt electrospinning process. We investigated the influence of the draw ratio, the laser power, and the applied voltage on the structure and properties of laser-electrospun poly(ethylene terephthalate) fibers, specifically, their birefringence, boil-off shrinkage, and mechanical properties. We found that when the draw ratio was increased, the birefringence and the boil-off shrinkage of electrospun fibers increased, while their natural draw ratio and elongation at break decreased. In addition, when either the laser power or the applied voltage was increased, the birefringence decreased, whereas the natural draw ratio and elongation at break increased. The boil-off shrinkage also decreased with an increase in the draw ratio, but the laser power and applied voltage mainly influenced for lowbirefringence region.
\end{abstract}

(Received 14 May, 2009; Accepted 12 June, 2009)

\section{Introduction}

Electrospinning is a method for producing ultra-fine fibers by drawing a polymer solution or a polymer melt by an electrostatic force. Electrospinning has a long history : the first patent was applied for in 1934 [1] and production of ultra-fine fibers with diameters of less than $1 \mu \mathrm{m}$ was first reported in 1971 [2]. In recent years, the technique has again been attracting attention with the increase in the demands made on ultra-fine fibers from various materials. However, most studies on electrospinning focus on electrospinning from polymer solution, which has the disadvantage that it requires a solvent, increasing both the production cost and the burden on the environment. In recent years, to overcome this disadvantage, so-called "melt electrospinning" has been studied [3-7]. Laser-electrospinning is one melt electrospinning method that involves heating the fiber material rapidly by laser irradiation. The rapid heating provided by laser irradiation reduces the viscosity of the material dramatically, and the material is instantaneously drawn by the electrostatic force without being thermally degraded.

Laser-electrospinning originates from the flow drawing method. Two drawing states, neck drawing and flow drawing, are generally observed in the continuous drawing process. Neck drawing leads to orientationinduced crystallization by generating a high drawing stress. In contrast, in flow drawing the fiber is drawn at a low drawing stress at a high temperature. In the flow drawing process, very little molecular orientation or crystallization is produced because of the low drawing stress. Whereas in neck drawing the draw ratio is limited to about 10 because of orientation-induced crystallization, there is no theoretical limit to the draw ratio for flow drawing. Therefore, flow drawing has a high potential to produce ultra-fine fibers. Unfortunately, the flow-drawing state tends to be unstable, because the fiber is drawn with decreasing viscosity. In particular, it generates a lower drawing tension and a longer deformation region than neck drawing. Therefore, flow drawing with a high draw ratio is difficult to achieve in practice when using a heattransfer heating method such as roll drawing. In 2002, Ohkoshi et al. reported laser flow drawing that overcomes this problem [8,9]. Laser flow drawing avoids instability by using rapid, uniform laser heating to greatly shorten the heating region. Consequently, using this method a fiber can be flow drawn at a high draw ratio and an ultrafine fiber having a diameter of about $1 \mathrm{~mm}$ can be produced $[10,11]$. However, instability cannot be fully avoided only by laser heating. In recent years, more stable flow drawing methods have been developed by combining laser heating with airflow [12] or electric force. Flow drawing stabilized by electrostatic force is known as "laser-electrospinning".

There have been several studies of laser- 
Table 1 Laser-electrospinning conditions, fiber diameter and mechanical properties.

\begin{tabular}{|c|c|c|c|c|c|c|c|c|c|c|c|}
\hline \multirow[t]{2}{*}{$\begin{array}{c}\text { Sample } \\
\text { No. }\end{array}$} & \multirow[t]{2}{*}{$\begin{array}{l}\text { Draw } \\
\text { Ratio }\end{array}$} & \multirow{2}{*}{$\begin{array}{c}\text { Laser } \\
\text { Power } \\
\text { /W }\end{array}$} & \multirow{2}{*}{$\begin{array}{c}\text { Applied } \\
\text { Voltage } \\
\text { /kV }\end{array}$} & \multicolumn{2}{|c|}{$\begin{array}{l}\text { Diameter } \\
/ \mu \mathrm{m}\end{array}$} & \multicolumn{2}{|c|}{$\begin{array}{c}\text { Tensile } \\
\text { Strength } \\
/ \mathrm{GPa}\end{array}$} & \multicolumn{2}{|c|}{$\begin{array}{l}\text { Breaking } \\
\text { Elongation }\end{array}$} & \multicolumn{2}{|c|}{$\begin{array}{c}\text { Natural } \\
\text { Draw Ratio }\end{array}$} \\
\hline & & & & Ave. ${ }^{*}$ & S.D. ${ }^{* *}$ & Ave. & S.D. & Ave. & S.D. & Ave. & S.D. \\
\hline 1 & \multirow{12}{*}{100} & \multirow{4}{*}{5} & 0 & 18.1 & 0.5 & 0.19 & 0.03 & 3.6 & 0.5 & 1.9 & 0.2 \\
\hline 2 & & & 5 & 18.1 & 0.5 & 0.18 & 0.03 & 4.0 & 0.5 & 2.2 & 0.2 \\
\hline 3 & & & 10 & 18.1 & 0.6 & 0.19 & 0.02 & 4.5 & 0.5 & 2.3 & 0.2 \\
\hline 4 & & & 15 & 18.0 & 0.5 & 0.19 & 0.03 & 3.6 & 1.0 & 2.0 & 0.5 \\
\hline 5 & & \multirow{4}{*}{5.5} & 0 & 17.1 & 0.5 & 0.18 & 0.04 & 4.3 & 0.5 & 2.5 & 0.2 \\
\hline 6 & & & 5 & 17.7 & 0.3 & 0.19 & 0.01 & 4.3 & 0.4 & 2.3 & 0.1 \\
\hline 7 & & & 10 & 17.8 & 0.6 & 0.19 & 0.02 & 4.4 & 0.6 & 2.3 & 0.2 \\
\hline 8 & & & 15 & 18.0 & 0.5 & 0.18 & 0.03 & 3.0 & 1.0 & 1.7 & 0.6 \\
\hline 9 & & \multirow{4}{*}{6} & 0 & 16.6 & 0.4 & 0.21 & 0.02 & 4.8 & 0.6 & 2.6 & 0.4 \\
\hline 10 & & & 5 & 17.4 & 0.5 & 0.17 & 0.02 & 4.9 & 0.6 & 2.6 & 0.3 \\
\hline 11 & & & 10 & 18.0 & 1.1 & 0.15 & 0.02 & 4.9 & 0.5 & 2.9 & 0.2 \\
\hline 12 & & & 15 & 17.5 & 0.4 & 0.19 & 0.02 & 3.8 & 1.1 & 2.3 & 0.4 \\
\hline 13 & 200 & 6 & 15 & 14.2 & 0.5 & 0.18 & 0.03 & 2.4 & 0.5 & 1.2 & 0.3 \\
\hline 14 & 300 & 6 & 15 & 12.3 & 0.7 & 0.18 & 0.02 & 2.5 & 0.5 & 1.2 & 0.1 \\
\hline
\end{tabular}

*) Average

**) Standard deviation

electrospinning [4-7]. Ultra-fine poly (ethylene terephthalate) (PET) fibers with a diameter of $1.7 \mu \mathrm{m}$ have been produced [6]. Radhakrishnan et al. have reported that flow-drawn PET films have different network structures compared with conventional drawn PET films [13]. In this present paper, we investigate the effect of the spinning conditions on the structure and mechanical properties of laser-electrospun fibers.

\section{Experimental}

\subsection{Laser-electrospinning}

Fig. 1 shows a schematic diagram of the laser electrospinning system. As-spun PET ( IV=1.0 dL/g, Toray) fiber, which was $200 \mu \mathrm{m}$ in diameter, was fed at a rate of $4 \mathrm{~cm} / \mathrm{min}$ and was electrically charged at the nozzle. The viscosity of the fiber was reduced by laser

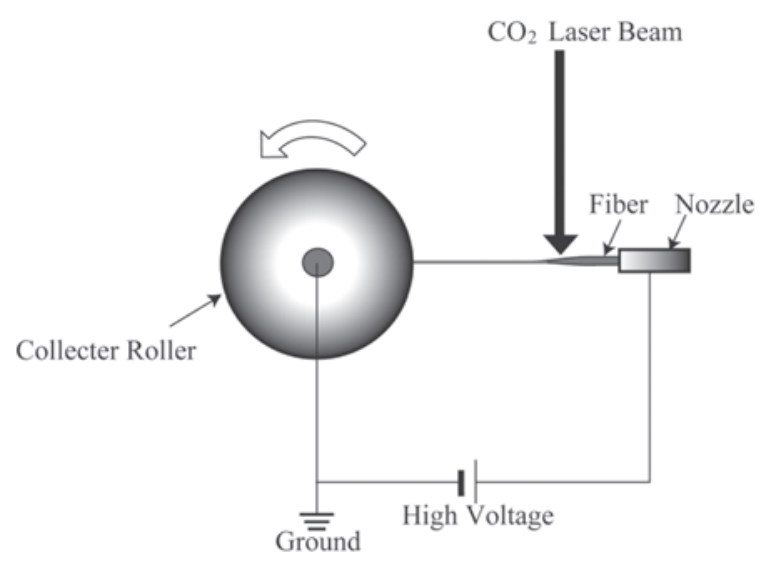

Fig. 1 Laser-electrospinning conditions, fiber diameter and mechanical properties. heating just behind the nozzle and the fiber was drawn by electrical force and by the take-up tension of the collector roller. The distance between the needle and the collector was $5 \mathrm{~cm}$. In this study, the electrospun fibers were produced while varying the applied voltage, the laser power, and the take-up speed of the collector roller. The electrospinning conditions for each sample are given in Table 1.

\subsection{Birefringence measurement}

The birefringence of the electrospun fibers were measured using a polarized microscope (BH-2, Olympus).

\subsection{Boil-off shrinkage measurement}

Samples with an initial length of $200 \mathrm{~mm}$ were immersed in boiling water for $30 \mathrm{~min}$ and the resultant length was measured. The shrinkage was calculated as follows :

$$
S=\frac{l_{0}-l}{l_{0}}
$$

where $S$ is the boil-off shrinkage, $l_{0}$ is the initial length, and $l$ is the length after shrinkage.

\subsection{Tensile testing}

The tensile properties of the laser electrospun fibers were measured at room temperature using a tensile tester (RTC-1250, Orientec Tensilon). The initial sample length was $40 \mathrm{~mm}$ and the extension rate was $100 \% \mathrm{~min}^{-1} ; 12$ measurements were performed for each sample.

\section{Results and discussion}

Table 1 shows the laser electrospinning conditions, 
fiber diameter, mechanical properties, and natural draw ratio of the samples obtained in this study. In all conditions, it was possible to spin continuously for at least about three minutes. When a laser power of $5 \mathrm{~W}$ or less was used, the fiber broke during electrospinning, and when a laser power of $6 \mathrm{~W}$ or more was used, polymer degradation was clearly seen. When a voltage was applied, an electric current flowed between the needle and the collector. When a voltage of $15 \mathrm{kV}$ was applied, a pulsed current, which was several times greater than the mean value, was repeatedly observed (Fig. 2(b)). When a voltage of $20 \mathrm{kV}$ was applied, the mean current became high and the fiber broke. Furthermore, for the applied voltage of $15 \mathrm{kV}$, the stress-strain curves of electrospun fibers show a lot of variation (Fig. 3(b)). The mechanical properties (elongation and natural draw ratio) also vary considerably. These results indicate that the electric field becomes unstable by the atmospheric discharge when the applied voltage exceeds $15 \mathrm{kV}$.

The dependences of the birefringence and the boiloff shrinkage on the draw ratio are shown in Fig. 4, and the dependences of the natural draw ratio and the elongation on the draw ratio are shown in Fig. 5. As the draw ratio increases, the birefringence and the boil-off shrinkage increase and the natural draw ratio and
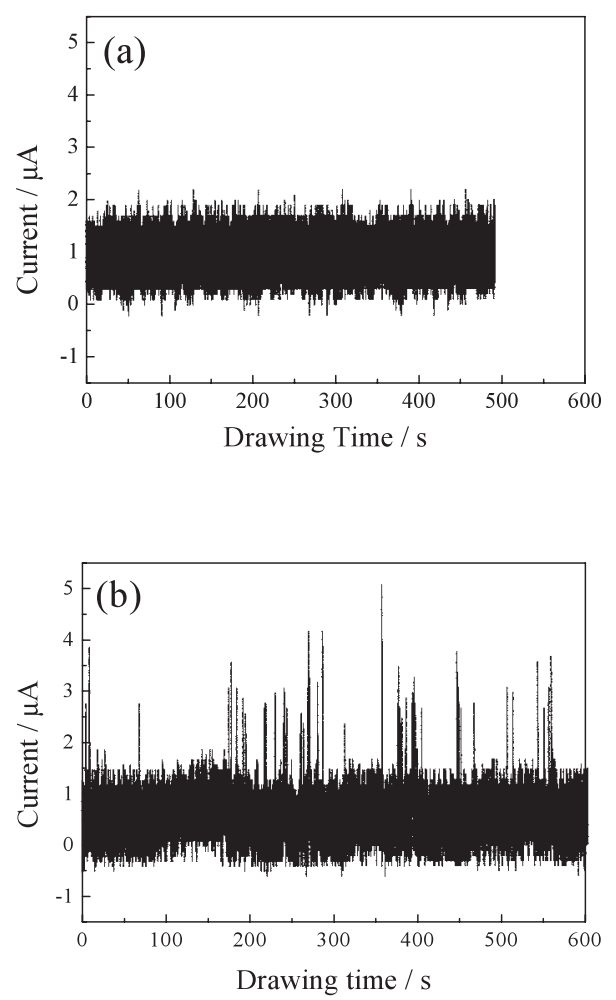

Fig. 2 Electrical current monitored during electrospinning for a laser power of $6 \mathrm{~W}$ and a draw ratio of 100 . The applied voltage is (a) $5 \mathrm{kV}$ (sample 9) and (b) $15 \mathrm{kV}$ (sample 12). elongation decrease. The true strain between the endpoint of natural drawing region and the breaking-point remains almost constant. This result implies that the samples have almost the same network structures [13].

Next, the dependences of the birefringence on laser power and applied voltage and the dependences of the boil-off shrinkage on laser power and applied voltage of laser electrospun fibers drawn to 100 times are shown in Figs. 6 and 7. Fig. 6 shows that the birefringence decreases when the laser power is increased. In addition, the birefringence decreases with an increase in the applied voltage at low laser powers, while the relationship between the birefringence and applied voltage is not clear at high laser powers. In contrast, Fig. 7 shows that the boil-off shrinkage decreases with an increase in the applied voltage at high laser powers, while the applied voltage has no observable effect on the boil-off shrinkage at low laser powers. The tensile strength of the laser electrospun fibers is not influenced by the applied voltage or the laser power, but the natural draw ratio of the fiber increases with an increase in the laser power, with the exception of the uneven fiber electrospun at a voltage of $15 \mathrm{kV}$ (Fig. 8). Furthermore, the natural draw ratio increases slightly with an increase in the applied voltage (Fig. 9).

Based on these results, we discuss the influence of
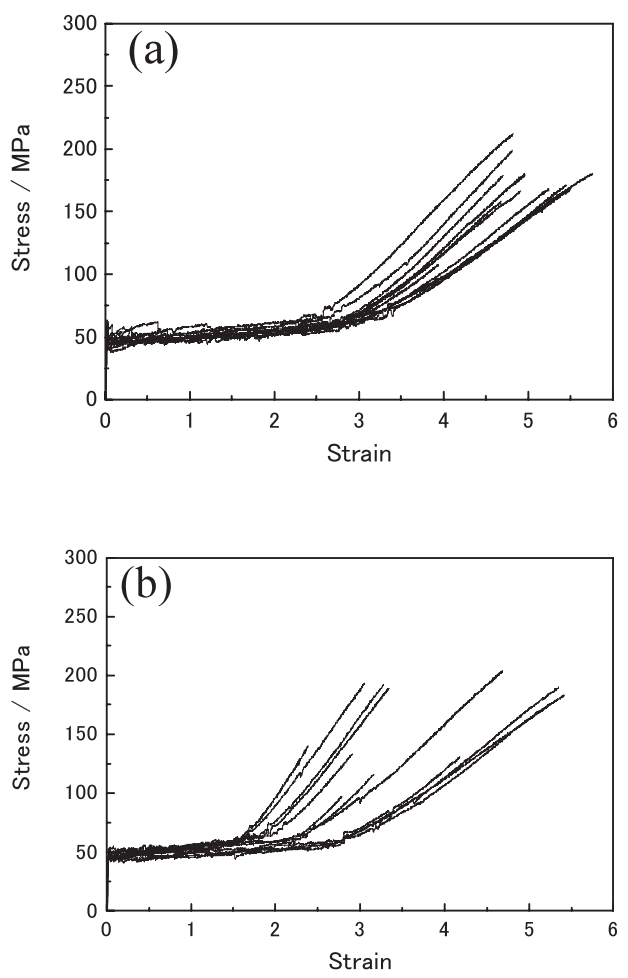

Fig. 3 Stress-strain curves for the fiber obtained by electrospinning. The spinning conditions are the same as those for Fig. 2. 


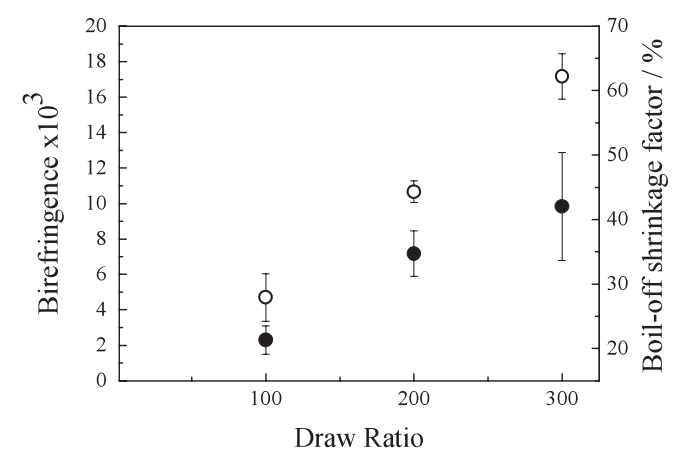

Fig. 4 The birefringence (O) and the boil-off shrinkage $(\bigcirc)$ of electrospun fibers plotted against the melt draw ratio (samples 12-14). The error bars represent the standard deviation.

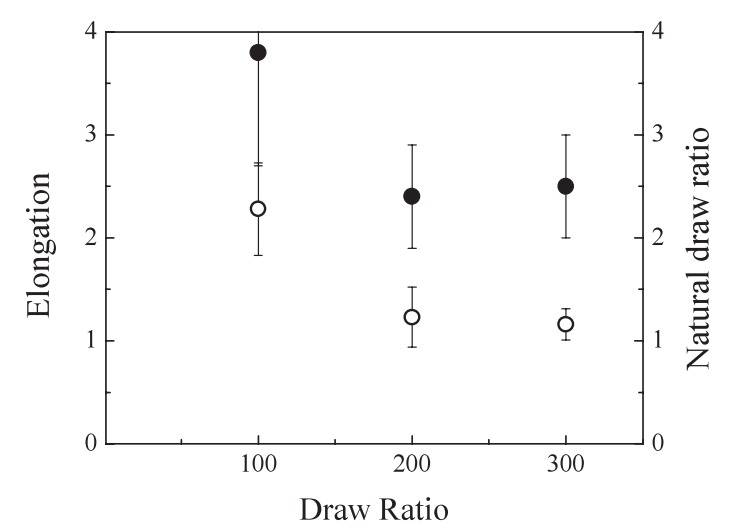

Fig. 5 The elongation at break (O) and the natural draw ratio $(\bigcirc)$ of electrospun fibers plotted against the melt on draw ratio (samples 12-14 in Table 1). The error bars represent the standard deviation.

the applied voltage and the laser power on the fiber structure in laser electrospinning. When a laser power is increased, the drawing temperature increases. Consequently, the fiber tension decreases with a decrease of polymer viscosity. On the other hand, the fiber tension also decreases with an increase in the applied voltage. This phenomenon can be explained as follows. The fiber is drawn three forces: the electrostatic repulsion generated by the electrical charge, the electrostatic attraction due to the electric field, and the take-up tension applied by the collector roller. The electrostatic repulsion decreases with leaving from the nozzle because the electrical charge decreases due to electrical discharge and the charge density decreases as the fiber becomes thin. On the other hand, the tensile stress produced by the take-up tension increases with distance from the nozzle and fiber thinning because the stress is in inversely proportional to the cross-sectional area of the fiber. Thus, the electrical charge influence the fiber mainly near the point where deformation starts. Therefore, the applied voltage decreases the deformation by the take-up tension near the

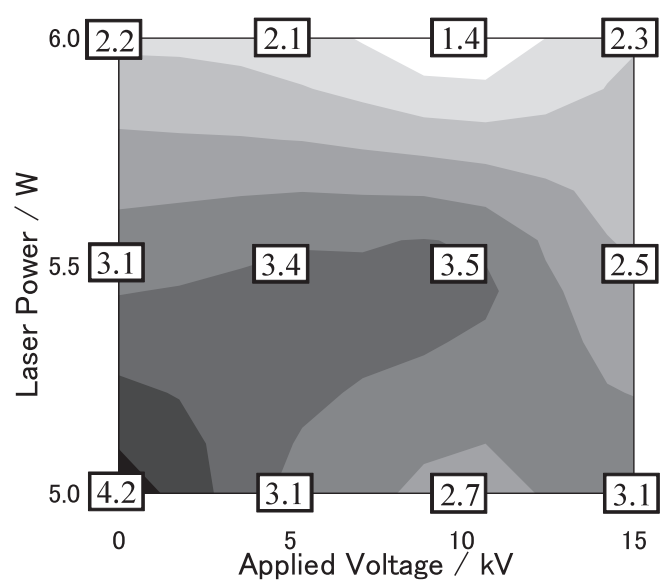

Fig. 6 Dependence of the birefringence on laser power and applied voltage. The birefringence was measured for the electrospun fibers spun under the conditions shown in the figure.

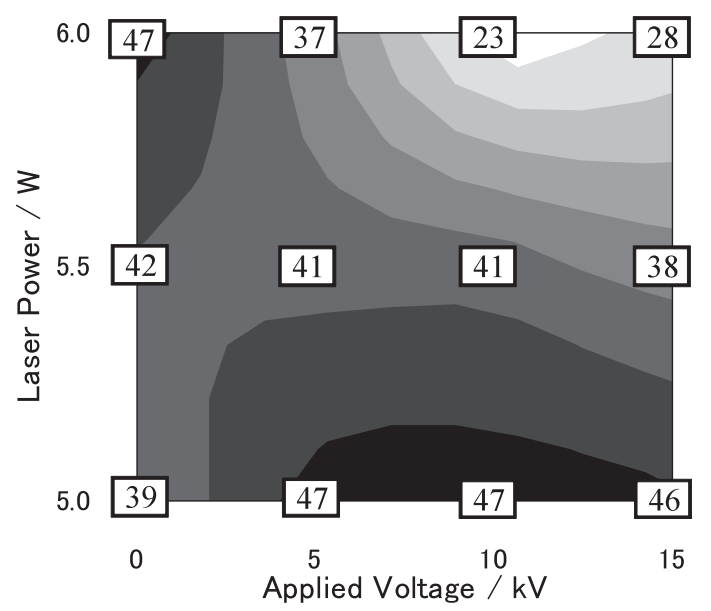

Fig. 7 Dependence of the boil-off shrinkage on laser power and applied voltage. The boil-off shrinkage was measured for the electrospun fibers spun under the conditions shown in the figure.

solidification point and the stress at the solidification point. When the stress at the solidification point is decreased, the birefringence and the boil-off shrinkage decrease and the natural draw ratio and elongation increase. The experimental results confirm these tendencies. The fibers produced in this study have low molecular orientations, so that the stress-optical law is applicable. Therefore, the decrease in the birefringence is directly related to the reduction in the drawing stress. The trend of the boil-off shrinkage is qualitatively similar to that of the birefringence, but the boil-off shrinkage mainly changes at low birefringence region. The quantitative difference between the trend of birefringence and boil-off shrinkage seems to be caused by the change in number of entanglement. The boil-off shrinkage is decided by the strain in the molecular chain between the entanglement points. That is, the birefringence is almost 


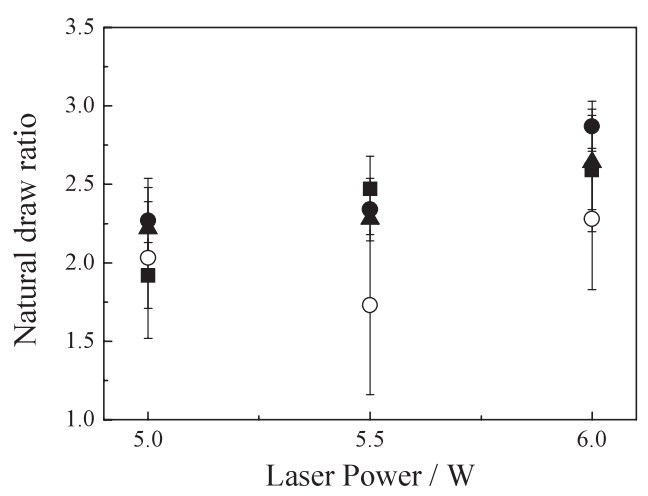

Fig. 8 Dependence of the natural draw ratio on laser power. The melt draw ratio of all samples is 100 and the laser power is $\boldsymbol{\square}: 5 \mathrm{~W}, \boldsymbol{\Delta}: 5.5 \mathrm{~W}$, and $: 6 \mathrm{~W}$.

proportional to the drawing stress at the solidification point, whereas the boil-off shrinkage depends on the amount of deformation of the molecular chain. As a result, if the drawing stress is reduced while keeping the draw ratio constant, the number of entanglement should be decreased. For the case, birefringence decreases proportionally to the solidification stress, whereas the boil-off shrinkage does not decrease so much because the whole molecular chain cannot be relaxed during the short relaxation time.

\section{Conclusions}

The influence of the draw ratio, the laser power, and the applied voltage on the structure and properties of laser-electrospun PET fibers, specifically, their birefringence, boil-off shrinkage, and mechanical properties were investigated. The results show that draw ratio strongly affects the birefringence, the boil-off shrinkage, the natural draw ratio, and the elongation at break. Increasing either the laser power or the applied voltage decreases the birefringence while it increases the natural draw ratio and elongation at break. The trend of the boil-off shrinkage is qualitatively similar to that of the birefringence, but the boil-off shrinkage mainly changes at low birefringence region.

\section{Acknowledgements}

This work was supported by a project for "Creation of Innovation Centers for Advanced Interdisciplinary Research Areas (Shinshu University)” in Special

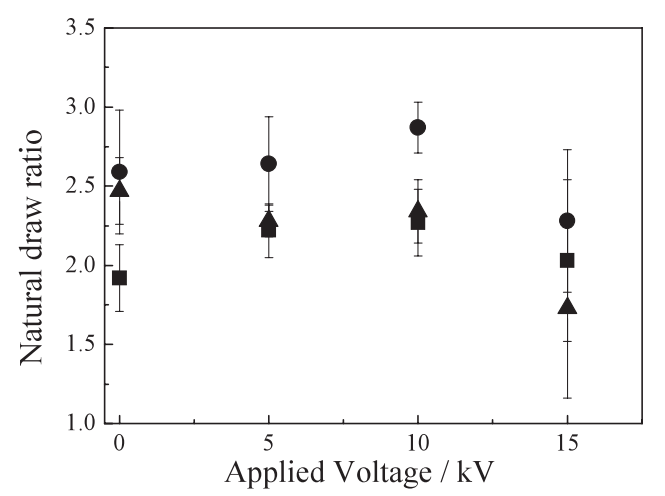

Fig. 9 Dependence of the natural draw ratio on applied voltage. The melt draw ratio of all samples is 100 and the applied voltage is $\mathbf{\square}: 0 \mathrm{kV}, \boldsymbol{\Delta}$ : $5 \mathrm{kV}, \mathrm{O}: 10 \mathrm{kV}$, and $\bigcirc: 15 \mathrm{kV}$.

Coordination Funds for Promoting Science and Technology from the Ministry of Education, Culture, Sports, Science and Technology of Japan. We also acknowledge the support of Global COE program from the Ministry of Education, Culture, Sports, Science and Technology of Japan.

\section{References}

1. A. Formhals, US Patent, 1975504 (1934).

2. P. K. Baumgarten, Journal of Colloid and Interface Science, 36, 71 (1971).

3. Z. Huajun, B. G. Thomas, L. J. Young, Polymer, 47, 7497 (2006).

4. N. Ogata, G. Lu, T. Iwata, S. Yamaguchi, K. Nakane, T. Ogihara, J. Appl. Polym. Sci., 104, 1368 (2007).

5. N. Ogata, S. Yamaguchi, N. Shimada, G. Lu, T. Iwata, K. Nakane, T. Ogihara, J. Appl. Polym. Sci., 104, 1640 (2007).

6. N. Ogata, N. Shimada, S. Yamaguchi, K. Nakane, T. Ogihara, J. Appl. Polym. Sci., 105, 1127 (2007).

7. M.Takasaki, H. Fu, K. Nakata, Y. Ohkoshi, and T. Hirai, Sen'i Gakkaishi, 64, 29 (2008).

8. Y. Ohkoshi, JP 115117 (2002).

9. Y. Ohkoshi, J. Watanabe, W. Okumura, Y. Gotoh, M. Nagura, Sen'i Gakkaishi, 58, 182, (2002).

10. A. Suzuki, Kobunshi, 52, 833, (2003).

11. A. Suzuki, N. Mochizuki, J. Appl. Polym. Sci. 88, 3279, (2003).

12. A. Suzuki, M. Kishi, Polymer, 48, 9, 2729 (2007).

13. J. Radhakrishnan, V. B. Gupta, J. Macromol. Sci.Phys., B32(2), 243 (1993). 